\title{
A tunable, microfluidic filter for clog-free concentration and separation of complex algal cells
}

\author{
E.J. Mossige ${ }^{1}$, B. Edvardsen ${ }^{2}$, A. Jensen ${ }^{3}$, and M.M. Mielnik ${ }^{4}$ \\ ${ }^{1}$ Department of Chemical Engineering, Stanford University, \\ Shriram Center, 443 Via Ortega 129, Stanford, CA 94305, \\ mossige@stanford.edu, Also at: Trilobite Microsystems A/S, \\ Torridalsveien 21A, 4630 Kristiansand, Norway \\ ${ }^{2}$ Section for Aquatic biology and toxicology, Department of \\ Biosciences, University of Oslo (UiO), P.O. Box 1053 Blindern, \\ N-0316 Oslo, Norway \\ ${ }^{3}$ Department of Mathematics, University of Oslo (UiO), P.O. Box \\ 1053 Blindern, N-0316 Oslo, Norway \\ ${ }^{4}$ Department of Microsystems and Nanotechnology (MiNaLab), \\ SINTEF Digital, , P.O. Box 124 Blindern, N-0314 Oslo, Norway
}

\begin{abstract}
An inherent problem with microfluidic filters is the tendency to clog, especially when applied to cells due to their geometrical complexity, deformability, and tendency to adhere to surfaces. In this work, we handle live algal cells of high complexity without signs of clogging, achieved by exploiting hydrodynamic interactions around trilobite-shaped filtration units. To characterize the influence of cell complexity on the separation and concentration mechanisms, we compare the hydrodynamic interactions to those of synthetic, rigid microparticles. We discover that simple rolling along the filter structures, which prevents clogging for particles, cannot be applied to cells. Instead, we find that inertial effects must be employed to minimize the filter-interactions and that this modification leads to only a minor reduction in device performance.
\end{abstract}

\section{Introduction}

A well established field in microfluidics is particle enrichment and fractionation [Beech et al., 2012, Lenshof and Laurell, 2010, Sajeesh and Sen, 2014], with many promising technologies such as filtration [Chen et al., 2014, Maria et al., 
2015, Jiang et al., 2018], deterministic lateral displacement [Huang et al., 2004, Loutherback et al., 2010, Liu et al., 2010, McGrath et al., 2014], inertial microfluidics [Godino et al., 2015, Hood et al., 2016, Ghadami et al., 2017], and centrifugation [Morijiri et al., 2013, Al-Faqheri et al., 2017]. Combinations of the above mentioned methods have also been demonstrated [Marchalot et al., 2014, Jiang et al., 2016, Prabhakar et al., 2015, Kim et al., 2009]. Owing to the ease of operation and scalability [Dijkshoorn et al., 2017], industrial applications range from algal harvesting [Barros et al., 2015, Olgae, 2015], where high throughputs are essential, to more conventional lab-on-a-chip applications such as diagnostics [Nam et al., 2016, Warkiani et al., 2015, Maria et al., 2017]. However, most of these technologies are developed to manipulate synthetic microspheres or simple, rigid cells such as yeast cells, and the ability to handle particles with a high degree of complexity in shape, deformability and surface properties can therefore be limited.

For example, microfilters and cross-flow filters are commonly employed for concentration of sphere-like, fixed cells, but fibers and flagella of many living microorganisms including algal cells dramatically increase the risk of clogging due to agglomeration of biomaterial and fouling near the filter structures [Babel and Takizawa, 2010]. However, by utilizing hydrodynamic interactions to increase the apparent particle size, particles comparable in size to the pores can be directed away from the filter structures, which greatly reduces the tendency to clog. The most widely used technique to increase the apparent particle size is rotation. Sugaya et al. [2011] utilized velocity shear near the channel wall in a hydrodynamic filter to induce a tumbling mode for rods, which increased their virtual size. However, since rotation is most efficient in the absence of inertia, the achievable throughput of this method can be limited.

In a popular alternative to microfiltration called deterministic lateral displacement, cells are displaced laterally by the use of pillar obstructions. This method shows promising behavior for blood cell enrichment and for dewatering of micro algae, but alignment with pillar structures reduces the degree of lateral drift which, in turn, reduces the separation efficiency [Beech et al., 2012]. To hinder the alignment, Holm et al. [2011] suggested to use channels that were shallower than the diameter of disk-shaped blood cells, but reductions in the channel depth can lead to high pressure losses and hence restricts the achievable throughput. In addition, low velocity zones downstream of pillars can lead to agglomeration.

As an alternative to the above mentioned techniques, the technology presented here instead employs arrays of trilobite-shaped, cross-flow filtration units (Figure 1) and was exploited by Dong et al. [2011] to enrich cancer cell with concentration ratios as high as 4 . However, the throughput was only $2 \mathrm{ml} / \mathrm{min}$ and the applicability of this study to real life situations is limited since only fixed (dead) cells were used. In a more recent work, Honsvall et al. [2016] used the same trilobite-filter to harvest both live and fixed micro algae cells and reported successful enrichment reported for simple, rigid, sphere-like cells. However, attempts to concentrate complicated, non-spherical or flexible cells were unsuccessful because these either slipped through or clogged the filtration 
channels.

We have previously used a simplified version of the trilobite-filter to demonstrate how hydrodynamic interactions between particles and tunable flow fields can be utilized to improve concentration and filtration performance and to hinder clogging [Mossige et al., 2016]. By means of flow visualizations and velocimetry measurements $(\mu \mathrm{PIV})$, we identified optimal flow conditions. In our most recent article [Mossige et al., 2018], we implemented these flow conditions for size-based concentration and separation of synthetic microspheres with throughputs as high as $32 \mathrm{ml} / \mathrm{min}$. Two distinctively different separation regimes were reported based on particle size and degree of inertia.

This article extends previous works by demonstrating concentration of complex, live algal cells of varying size, shape and deformability. While maximizing concentration ratios, we show that clogging is avoided and demonstrate enrichment of cells which are smaller than the filter pores. These achievements are realized by utilizing inertial effects, which are rarely exploited in microfluidic filters. We visualize cell trajectories by fluorescence imaging to characterize the interaction between algae, filter units and flow fields. By comparison with results obtained using synthetic microspheres, we isolate the effect of shape, deformability and surface properties on the separation dynamics and device performance. Finally, we utilize the difference in separation mode and inertia to separate flexible, rod-like cells from rigid, disk-like cells.

\section{Concentration and separation principle for syn- thetic particles}

Figure 1 shows how hydrodynamic principles around trilobite-shaped filtration units are utilized for concentration and separation. The filtrate flow is guided through slits $(25 \mu \mathrm{m})$ between turbine blade-shaped pillars, while the separation particles, which are visualized by streaklines, are guided around the trilobites and directed onto a different outlet, where the concentrate is collected. These particles interact with the pillars, however the strong shear flow in the accelerated flow region between trilobites induces particle rotation, which prevents clogging.

The combination of the incoming feed flow and the suction flow through the filtrate outlets creates a saddle point of converging streamlines, see Figure 1 (b). In Mossige et al. [2016], we found that clogging can be prevented and that concentration ratios are maximized when the saddle point is positioned directly downstream of the trilobite unit, as is the situation in (b). Since the flow has to go around the trilobites, a filtration layer with thickness $T$ is formed, and in Mossige et al. [2018], we showed that clogging is avoided when the flow is tuned so that $T$ is made smaller than the particle. As illustrated in (b), this flow configuration can be exploited to concentrate large (red) spheres and simultaneously separate them from green ones, which are smaller than the slits, because the latter type of particle simply follows the flow of filtrate. 
By utilizing flow inertia, which is attained by increasing the feed flow rate, $Q$, it is possible to concentrate particles which are smaller than the slit width, and this principle is shown in (c) and in (d). Owing to inertial effects in (c), the particle cannot follow its associated streamline; instead, it bumps against the filtration pillars and is thus carried downstream without entering the filtration slits (see schematic in (d)). This phenomenon is similar to the situation experienced by many frustrated golf players when the golfball has too much inertia to enter the hole without bouncing off. Farther downstream, when the 'golfball' (green particle) approaches the trilobite rear end, the combination of high fluid inertia and streamline curvature leads to lateral migration away from the filter structures. Both golfball-bouncing and lateral migration serve to increase the apparent (hydrodynamic) particle diameter above the size of the slit widths because particles which are smaller than the slits are prevented from entering the filtrate. A drawback of this method compared to the low velocity rolling used to concentrate the large red spheres in (b) is that the concentration ratio is reduced, due to the necessary reduction of the filtrate layer thickness $T$.

The array of 13 side-by-side trilobite separation units on the microfluidic chip used in this work (d) are distributed across the $10 \mathrm{~mm}$ wide main channel (30 $\mathrm{mm}$ long and $90 \mu \mathrm{m}$ deep). Each trilobite is $460 \mu \mathrm{m}$ wide and $1155 \mu \mathrm{m}$ long. The filtrate liquid flows through the trilobite outlets and is directed out of the device through another channel underneath the row of trilobites. The inlet, 'IN', and concentrate outlet, 'OUT', are connected to the main channel via distribution channels and the chip is sealed with a $0.30 \mathrm{~mm}$ thick glass slide.

\section{Experiments}

\subsection{Experimental setup}

The two parameters used to modify the flow fields and manipulate the migration of particles are the feed and filtrate flow rates, which are denoted as $Q$ and $Q_{f}$, respectively. The filtrate flow layer, which has thickness $T$ and dictates the cut-off size, is manipulated by tuning the ratio of these two flow rates. An increase in the relative amount of filtrate to feed fluid, $Q_{f} / Q$, leads to a thickening of $T$, while a decrease leads to a thinning of $T$. A user controlled pressure system (MFCS-EZ, Fluigent) is employed to modify the flow rates and these are controlled by using laboratory scales (ML 303T, Mettler Toledo) to weigh the accumulated liquid in respective reservoirs. The flow fields are easily reproduced because the flow rates are proportional to the reservoir pressures, and the maximum achievable throughput with our system was $32 \mathrm{ml} / \mathrm{min}$. Any signs of clogging were detected by continually monitoring the flow rates and by visually inspecting the filter during the experiments.

By illuminating particles by green light, produced by passing white light through an excitation filter (ZET532/10x, Chroma), they become fluorescent and emit a strong optical signal against the dark background. A beam splitter (z532rdc, Chroma) and an emission filter (HQ 580/60, Chroma) serve to further 
improve the optical signal by filtering out the excitation wavelengths. Live algae are intrinsically fluorescent, which allows us to study their dynamics without doping them with fluorescent material. Since doping leads to algal death, which dramatically changes their mechanical properties (for example, they generally become stiffer and more dense), it is preferred to avoid it if possible.

By capturing long exposure images of algal cell and particle trajectories, we characterize the hydrodynamic interactions and separation mechanisms. We use an upright microscope (BX43, Olympus) in combination with a 10x-objective (Olympus PlanC N 10x/0.25) and a CCD-camera (pco.4000, pco) for imaging and we determine the cell and particle concentrations by counting the number of cells inside equally sized drops $(2 \mu \mathrm{l})$ deposited onto glass slides. We calculate the concentration ratios as the number of particles in the concentrate fluid divided by the number of particles in the feed, measured per unit volume of fluid.

There is a $10 \%$ uncertainty in the calculation of the enrichment ratios performances, where the main error contribution comes from uncertainties in determining the particle and cell concentrations. Temporal fluctuations and mass flow variations between experiments count for only $1 \%$ of the uncertainty, due to repeatable and stable flow fields.

\subsection{Algal cells used in the experiments}

The algal cells, see Table 1 and Figure 2, are chosen to maximize the variability in size, shape and deformability. The two marine species, Prorocentrum minimum (M1) and Protoceratium reticulatum (M2), are nearly spherical; M1 (13 by $17 \mu \mathrm{m})$ is smaller than the $25 \mu \mathrm{m}$ filtration slits, and M2 (29 by $30 \mu \mathrm{m})$ is larger than the slits, while the two freshwater species, Cryptomonas rostratiformis (F1) and Micrasterias truncata (F2) have complex shapes; F1 is rod-shaped (19 by $41 \mu \mathrm{m})$ and F2 is disk-shaped (37 by $77 \mu \mathrm{m})$. Furthermore, M1, M2 and F2 are rigid, while $\mathrm{F} 1$ is flexible, and cell type $\mathrm{M} 1, \mathrm{M} 2$ and $\mathrm{F} 1$ are swimming organisms (for more information about the motility of cells, see the Appendix).

\subsection{Calibration using test particles}

Prior to the algae cells experiments, we verified the system for separation and concentration of rigid polymer beads (Cospheric LLC, CA, USA), called 'test spheres', with the following mean diameters: 21, 24, 32 and $69 \mu \mathrm{m}$, see Table 2 . Since these particles are neutrally buoyant in the immersion medium, which is DI-water, their hydrodynamic interactions with the flow fields are not influenced by density differences, which can give rise to additional inertial contributions. Live algal cells, on the other hand, are typically somewhat heavier than their immersion medium (Table 1), but since this density difference is typically only about 5\% [Kamykowski et al., 1992], these contributions are weak. Consequently, effects of density differences were well within the $\sim 10 \%$ measurement uncertainty of the experiments and thus comparison between particles and cells was feasible. 
By finding the minimum applied inflow that ensured concentration without clogging for each of the four test particle sizes, we identified maximum concentration ratios (CR). The results are presented in Table 2. By employing a least square fit to the four data points, $\mathrm{CR}$ was found to be proportional to the particle diameter squared and hence concentration efficiency increases rapidly with size. Test particles smaller than pores were concentrated by inertial migration, and spheres larger than pores were concentrated by low-velocity rolling along the filtration pillars. The applied flow rates that maximized CR served as reference for the subsequent testing with complex algal cells.

Long-range hydrodynamic interactions can increase the effective viscosity of the fluid. Since an increase in viscosity reduces particle inertia, which must be maintained to ensure maximized concentration performance (maximized $\mathrm{CR}$ ), high particle concentrations can reduce the device performance. By comparing flow fields obtained with test particles to flow fields obtained without using test particles, we found that adding particles did not alter the flow fields. This convenient result is mainly due to the low particle concentrations in the feed fluid $\left(<10^{-4}\right.$ by weight, see Table 2$)$, ensuring that long range hydrodynamic interactions between particles are negligible. For these calibration measurements, we performed streakline visualizations using $1 \mu \mathrm{m}$ tracers that follow the fluid flow accurately.

\section{Results}

\subsection{Cell enrichment}

M1: Figure 3 (a) and (b) show trajectories of the sphere-like M1-cells, which are visualized by streaklines. In (a), these small cells (13 by $17 \mu \mathrm{m})$ follow the flow of filtrate passively through the filtration slits because the velocity is too low to induce lateral drift, which is necessary for separation from the filtrate stream and hence to concentrate them. In (b), the velocity is increased to about $2 \mathrm{~m} / \mathrm{s}$, achieved by employing the maximum feed flow rate of $32 \mathrm{ml} / \mathrm{min}$. The velocity increase leads to lateral drift away from pillars in the downstream (curved) region of the trilobite filtration units due to increased inertia. However, a finite number of cells still slipped through the slits between the first pillars because the velocity was not sufficiently high to trigger the golfball-effect, which is used to bounce particles away from pillars and into the bulk flow.

M2: Figure 3 (c) shows the result of an attempt to concentrate the larger M2-cell (29 by $30 \mu \mathrm{m}$ ) by low-velocity rolling along pillars, which is the method used to concentrate large test particles. As is evident from the image, interactions between cells and pillar structures lead to clogging, especially in the upstream region. Hence, interactions with pillars resulting from attempts to roll the cells over filtration pillars must be avoided. In (d), the velocity is increased compared to the situation in (c). The velocity increase induced lateral

drift of cells, which, in turn, reduced the degree of pillar interactions and thus served to prevent clogging. 
F1: Figure 4 shows trajectories of the rod-like F1 cell. In (a), the cells simply follow the flow through the pillar slits because the velocity is too low to separate them from the filtrate stream. In (b), the velocity is increased, which lead to lateral migration across streamlines in the downstream region and hence to successful concentration of cells. However, during impact with pillars, these flexible cells deform instead of being deflected into the bulk flow by means of golfball-migration. Therefore, although high velocities were employed, there was still a finite amount of cells that slipped through the first filtration gaps, as is evident from the visualization in (b).

F2: Figure 5 (a) shows an attempt to concentrate disk-shaped F2-cells by low-velocity rolling over the filtration pillars, which is the separation technique used for large test particles. The applied flow rate, $10 \mathrm{ml} / \mathrm{min}$, is the same as was used to concentrate $69 \mu \mathrm{m}$ particles, which are comparable in size to the diameter of these disk-shaped cells $(77 \mu \mathrm{m})$. However, since these cells are pushed against the trilobite head, by a favorable pressure gradient near the stagnation point, they align with the wall and thus any tendency to roll is suppressed. An unfortunate result of the alignment is clogging of the first filter slit, since the cells slide along the trilobite, while passively following the filtrate flow. Although the clogged cell seen in the image prevents further clogging by deflecting other cells into the bulk stream, an increase in the inflow rate is required to remove clogging altogether.

In (b), the flow rate is increased to $Q=18 \mathrm{ml} / \mathrm{min}$, which is the flow rate used to concentrate $32 \mu \mathrm{m}$ test spheres, which are comparable in size to the cell thickness $(37 \mu \mathrm{m})$. The velocity increase from (a) to (b) leads to a strong shear

flow as well as to an adverse pressure gradient near the first filtration pillars (see also the cartoon in (d)). The combination of these two mechanisms induces inplane rotation (forward flipping), which results in a new orientation of the cell, where the largest cell face is now pointing against the incoming flow. A strong lift force resulting from the new orientation pushes the cell away from pillars and into the accelerated flow region between trilobites. Further downstream, the cell experiences out-of-plane rotation and resume the orientation it held upstream of the trilobite unit.

Carefully conducted experiments showed that the velocity in (b) was higher than necessary to avoid clogging. Thus, clog prevention was possible with lower inflow rates than the employed $Q=18 \mathrm{ml} / \mathrm{min}$, and hence an increase in concentration ratio was possible. In (c), the critical inflow rate is used, $Q_{\text {critical }}=$ $14 \mathrm{ml} / \mathrm{min}$. With $Q_{\text {critical }}$, the velocities were still sufficiently high to induce inplane cell rotation, which is necessary to prevent clogging, however the rotation occurs farther downstream than in (b).

\subsection{Performance of cell enrichment}

Figure 6 (a) is a plot of the maximum achievable CR without clogging. The red dots are the data points obtained using test particles $(21,24,32$ and 69 $\mu \mathrm{m})$ and the solid line is a second order fit to the obtained four data points, showing excellent agreement $\left(R^{2}=0.9997\right)$. An attempt to achieve higher CR 
than is indicated by the solid line would result in clogging because it can only be achieved by reducing the inflow rate. The maximum achievable CRs for algae are indicated by image symbols and circles; images indicate longest algae dimension and circles indicate shortest dimension.

Comparisons of the hydrodynamic diameters of cells to the physical dimensions (width, length and diameter) enable evaluation of the effect of cell complexity, e.g. different shapes, on the concentration performance. For example, when the hydrodynamic diameter is larger than the physical dimensions, it means that the cell shape helps to increase the CR, while if the hydrodynamic diameter is smaller than the physical measures, then the opposite is true. By convention, the CR of an algal cell with hydrodynamic diameter of $a_{H}$ is exactly equal to that of a test sphere with diameter of $a$. The CR-curve was used to calculate $a_{H}$, by the method shown in (b), and the results are presented in (c). Comparisons between the virtual and physical dimensions of algae are presented in Table 3.

\subsubsection{Cells with simple shapes}

For sphere-like M2-cells (29 by $30 \mu \mathrm{m}$ ), the hydrodynamic diameter is smaller than the physical dimensions, which means that the CR is not as high as that of a rigid sphere of similar size. The reason for the efficiency deficit is that the M2-cell cannot be concentrated by low-velocity rolling over the filtration pillars as these types of interactions lead to clogging, which is most likely due to the particularly rough cell surface. To avoid clogging, the degree of pillar interactions must be reduced, and therefore, the M2-cell must be concentrated by lateral migration instead of rolling, which is attained by employing high flow velocities. Unfortunately, the required velocity increase necessary to obtain the sufficient amount of inertia leads to a slight reduction in CR, caused by the resulting thinning of the filtrate layer.

For the small M1-cell (13 by $17 \mu \mathrm{m}$ ), the hydrodynamic diameter is larger than the physical dimensions, which indicates that the cell complexity actually increases the concentration efficiency. However, this positive result should be read with some skepticism since it was found by extrapolation; the test sphere diameters used to create the CR-curve range from 69 to $21 \mu \mathrm{m}$, while the M1-cell is 13 by $17 \mu \mathrm{m}$, which is outside this range of particle sizes.

\subsubsection{Cells with complex shapes}

The hydrodynamic diameter of the rod-like F1-cell is $22.2 \mu \mathrm{m}$, and the similarity with the width $(19 \mu \mathrm{m})$ indicates that influences of the length $(41 \mu \mathrm{m})$ on the concentration mechanism are secondary, however they are not negligible. The similarity with the width is due to alignment with the upstream body of the trilobite, resulting from a favorable pressure gradient, causing the smallest cell length (the width) to face the incoming flow.

Also for the disk-shaped F2-cell, the concentration ratio is dictated by the shortest cell dimension, seen as the almost identical value of the width, $w$, and 
$a_{H Y D}$. Again, the reason for this similarity is alignment with the trilobite head, which causes the cell to slide along the wall, with the smallest cell face pointing against the direction of the flow. The alignment suppresses any tendency of the cell to flip or rotate to increase its hydrodynamic diameter beyond its width.

\subsection{Comparison with previous work and prospects of up- scaling}

As mentioned in the Introduction, higher concentration ratios than reported in Table 3 were demonstrated by Dong et al. [2011], using a modified version of our microfluidic device. However, the focus of our work is enrichment of complex cells without clogging, achieved by employing tunable flow fields, rather than maximization of concentration ratios. Another important difference between our works is the total number of employed trilobites. While Dong et al. [2011] made use of as much as 700 units, yielding a concentration ratio of 0.005 per trilobite, only 13 units were used in the current experiments, yielding a value of 0.09 for the same performance metrics. Thus, our work represents an improvement.

To further enhance the concentration performance of our design, one could simply add rows of trilobites. To compensate for the removal of fluid through the additional trilobite filter outlets, one could make use of a contracting main channel to maintain the velocities needed to prevent clogging.

Finally, owing to the simple chip layout, the technology has potential for parallelization. A substantial increase in throughput is therefore possible, which

enables implementation in industrial processes where several cubic meters of fluid are processed every minute. Industries where high throughputs are essential include micro algal harvesting and ocean tech industries with applications such as micro plastics removal.

\subsection{Cell sorting}

The results of cell enrichment using individual algal cells are strong indications that fractionation from a mixture of different species is possible. In order to test this hypothesis, we mixed large test spheres with small test spheres and performed fractionation. This initial testing was followed by sorting of real cells, and the results were then compared to study the effect of cell complexity on the fractionation performance.

\subsubsection{Calibration and validation with test spheres}

Figure 7 (a) shows a streakline visualization where 32 and $69 \mu \mathrm{m}$ spheres were successfully separated from $21 \mu \mathrm{m}$ spheres. With the employed feed flow rate, $Q=21 \mathrm{ml} / \mathrm{min}$, the velocities were sufficiently high to induce clog-preventing rolling over the pillars for $32 \mu \mathrm{m}$ spheres. Note that the filtration layer $T$ was also approximately $32 \mu \mathrm{m}$, which marks the clog-free cut-off size. Since the $69 \mu \mathrm{m}$ spheres were much larger than the cut-off and due to the increased 
inertia as compared to $32 \mu \mathrm{m}$ spheres, they were not able to follow streamlines. Consequently, they followed a more or less straight trajectory around the trilobite-shaped filtration units. In stark contrast to the large $69 \mu \mathrm{m}$ spheres, the smaller $21 \mu \mathrm{m}$ spheres accurately followed the flow because the velocities were too low to induce inertial migration across streamlines and away from the filter units. Hence, $21 \mu \mathrm{m}$ spheres were carried by the filtrate through the filtration slits. As a convenient result of the difference in inertia and separation mode for different particle sizes, small particles were effectively separated from their larger counterparts without signs of clogging.

Optimally, the velocities used for fractionation are sufficiently high to prevent clogging of large particles, yet small enough that inertial effects are negligible for small particles, allowing them to follow the filtrate flow passively. In the case of negligible inertia, there is no concentration of small particles, resulting in an equal number of particles in the filtrate, concentrate and feed solutions, measured per unit volume of fluid. The results obtained using rigid, simple test spheres show no measurable difference in particle concentration between inflow, concentrate and filtrate, which implies complete separation of particles $(100 \%$ efficiency).

A major strength of the reported separation technology and employed operation is that the filtrate flow is insensitive to changes in feed flow rate. As reported in Mossige et al. [2018], the filtrate flow is constant and equal to 2 $\mathrm{ml} / \mathrm{min}$ regardless of inflow rate, which is considered as high for microfluidic separators. With a volumetric concentration of particles of $10^{-4}$ in the feed, which is a common concentration, 40,000 particles of size $21 \mu \mathrm{m}$ are separated per minute.

\subsubsection{Fractionation of complex cells}

To verify that our geometry can be used to sort live algal cells of distinctively different shape, size and deformability, we mixed soft, rod-like F1-cells with rigid, disk-like F2-cells in the inflow and performed fractionation, see the streakline visualizations in Figure 7 (b). We applied the flow rate required to concentrate the latter type of cell by separation from the filtrate stream without clogging, namely $Q=14 \mathrm{ml} / \mathrm{min}$. Our results show that no clogging occurred and that there were no F2-cells in the filtrate, which is to be expected as these cells are larger than the slits.

The streakline visualization shows that the rod-shaped F1-cells followed the filtrate stream passively through the filtration slits, which is because the velocity is sufficiently low that inertial effects were small. However, due to some degree of pillar interactions caused by the fact that the cell is longer than the slit width (length is $41 \mu \mathrm{m}$ while slits are $25 \mu \mathrm{m}$ ), there were $13 \%$ fewer cells per unit volume in the filtrate than in the feed. Hence, the separation efficiency was 87 $\%$, which is comparable to the separation efficiency obtained using rigid spheres (100\% efficiency).

With 16,000 F1-cells per milliliter of feed fluid, a filtration flow rate of 2 $\mathrm{ml} / \mathrm{min}$ and a separation efficiency of $87 \%$, the fractionation rate is close to 
28,000 cells per minute. With smaller cells than the F1-cell, for example with the M2-cell, even higher fractionation rates can be expected due to an increased number of cells in the feed fluid and higher separation performance because these cells follow the flow more accurately.

\section{Conclusion}

A separation technology consisting of trilobite-shaped filters was used for enrichment and sorting of four types of live algae cells which were distinctively different in size, shape and deformability. The flow was tuned to maximize concentration ratios without clogging. Optimal flow conditions were identified for each cell type by means of streakline visualizations and controlled flow rate measurements.

Unlike synthetic microspheres, algal cells cannot be concentrated by lowvelocity rolling along the filter structures since these interactions lead to clogging. Instead, inertial flow fields are employed to achieve cross-streamline migration and golfball-bouncing away from the filters. For one of the species in our study, namely the sphere-like M2-cell, the high flow rate needed to obtain the sufficient amount of inertia to prevent clogging lead to a slight reduction in concentration ratio because of a reduction in the ratio of filtrate to feed fluid. However, for the remaining three cells types, including complex rod-shaped and disk-shaped cells, the obtained performances were comparable to that of synthetic particles, when evaluated by the shortest cell dimension.

Finally, differences in the degree of inertia and deformation during impact with pillars were utilized to sort large disk-shaped algal cells from small, flexible rod-like cells. The smaller rod-like cells were sorted at a rate of 28,000 cells per minute, conserving the excellent fractionation characteristics reported previously for synthetic microspheres.

\section{Acknowledgements}

This project was funded by Trilobite Microsystems A/S and The Research Council of Norway - project number 232148. Support was also received from the Norwegian micro- and nanofabrication Facility (NORFAB) infrastructure project. All measurements were conducted at the Hydrolab at the Department of Mathematics at the University of Oslo in Norway. Thanks to SINTEF MiNaLab for manufacturing the microfluidic chips, to Vladyslava Hostyeva at NIVA and Bente Edvarsen at Department of Biosciences, UiO, for providing the algal cultures and the growth media. Also, thanks to Rita Amundsen at Department of Biosciences, UiO, for overlooking the cultures. Thanks to our lab engineer Olav Gundersen for assistance with the pressure system and to Dag Dysthe at the Department of Physics (UiO) for lending us the camera, and thanks to Lailai Zhu in the Complex Fluids group at Princeton University for general feedback on the manuscript. 


\section{Appendix}

\subsection{Swimming behavior of algae}

The marine dinoflagellates (M1,M2) have two flagella and have a helical swimming pattern. Cryptomonas rostratiformis (F1) has two flagella, the forward with thin hairs is dragging the cell forward while erected. Figure 8 shows trajectories of the fastest swimmer used in this study, namely algae Protoceratium reticulatum (M2). Figure 8 (a) was obtained using a 4x-objective and Figure 8 (b) was obtained using a 10x-objective and the exposure time of both images was 5 seconds. Based on the length of the streaks, the swimming speed is estimated to $\sim 0.15 \mathrm{~mm} / \mathrm{s}$, which is negligible compared to the speed of cells in the flow field around the separation units $(\sim 2 \mathrm{~m} / \mathrm{s})$. Therefore, the swimming activity of cells used in this study does not influence the separation dynamics.

To be able to be stationary in the liquid without sinking or rising and to ease the task of swimming, the densities of the swimming organisms are only about 5\% higher than that of water. The F2-cell is not a swimmer, but its disk-shaped body enables migration across streamlines, which allows it to be easily transported by whirls and currents over large distances. To fully exploit this transportation method, this cell is also nearly neutrally buoyant.

\subsection{Algal cultures}

The microalgae used in this study were obtained from the Norwegian Culture Collection of Algae. The marine dinoflagellates Prorocentrum minimum (strain UIO 089) and Protoceratium reticulatum (strain UIO 232) were isolated from the Oslofjorden, Skagerrak, Norway, in 1986 and 2001 respectively. The freshwater microalgae Micrasterias truncata (strain NIVA-CHL 34) was isolated from River Storelva, Ringerike, in S. Norway in 1978 and Cryptomonas rostratiformis (strain NIVA-3/81) from Lake Helgetjernet in 1981.

The marine cultures were grown in the algal medium IMR $\frac{1}{2}$ medium [Eppley et al., 1967], supplemented with $10 \mathrm{nM}$ selenium [Edvardsen et al., 1990] with salinity of 25 PSU. The freshwater algal strains were grown in the medium Z8 [Kotai, 1972] or a modified Z8 medium. The media were sterilised by pasteurisation at $80^{\circ} \mathrm{C}$ for $20 \mathrm{~min}$. All four strains were grown at $16^{\circ} \mathrm{C}$ under fluorescence white light with an irradiance of approximately $50 \mu \mathrm{mol}$ photons $\mathrm{m}^{-2} \mathrm{~s}^{-1}$, and a 14h:10h light: dark cycle. They were grown as batch cultures in $5 \mathrm{~L}$ Erlenmeyer flasks and harvested in the exponential or beginning of stationary growth phase. 


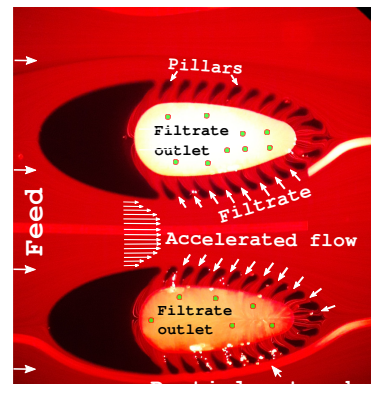

(a)

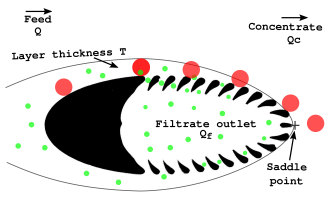

(b)

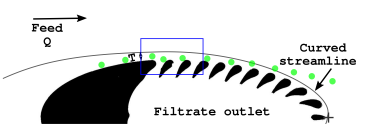

(c)

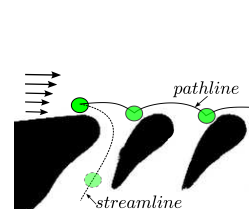

(d)

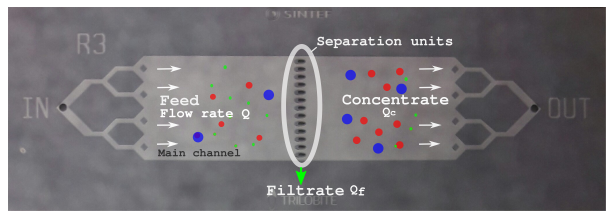

(e)

Figure 1: (a) Concentration of rigid microspheres by migration along two trilobite filter units. The bright streaks are the trajectories, obtained from longexposure images of fluorescent particles. (b) Simultaneous concentration of red spheres, which are larger than gaps, and separation from green spheres, which are smaller than gaps. Rolling along the pillars at relatively low velocities, which is induced by the filtrate shear layer, helps to prevent clogging of large particles. (c) Concentration of spheres smaller than slits by utilizing inertial effects. Upstream, inside the square, the so-called golfball-effect is used to prevent the particles from entering the slits and in the downstream region, lateral migration across the curved streamlines is used. These two mechanisms serve to increase the hydrodynamic diameter, which enables concentration of particles smaller than slits. Without inertia, these small particle would have followed the filtrate flow passively, making concentration impossible. (d): Close-up of the golfballeffect; without inertia, the particle would have followed its associated streamline through the filtration slits. (e) Separation microchip: The concentration and separation of large (red) spheres from small (green) spheres are performed by the separation units. 


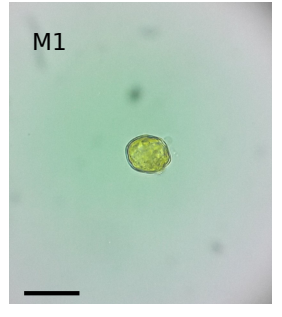

(a)

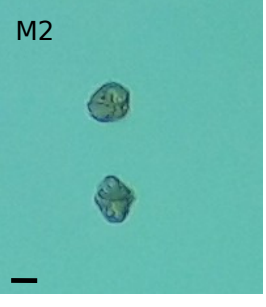

(b)

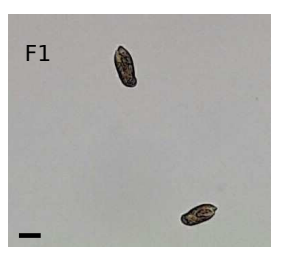

(c)

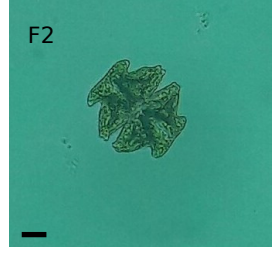

(d)

Figure 2: Algal cells used in this study (a) Prorocentrum minimum UIO (M1), (b) Protoceratium reticulatum UIO (M2), (c) Cryptomonas rostratiformis NIVA (d) Micrasterias truncata NIVA. Scale bar is $20 \mu \mathrm{m}$. 


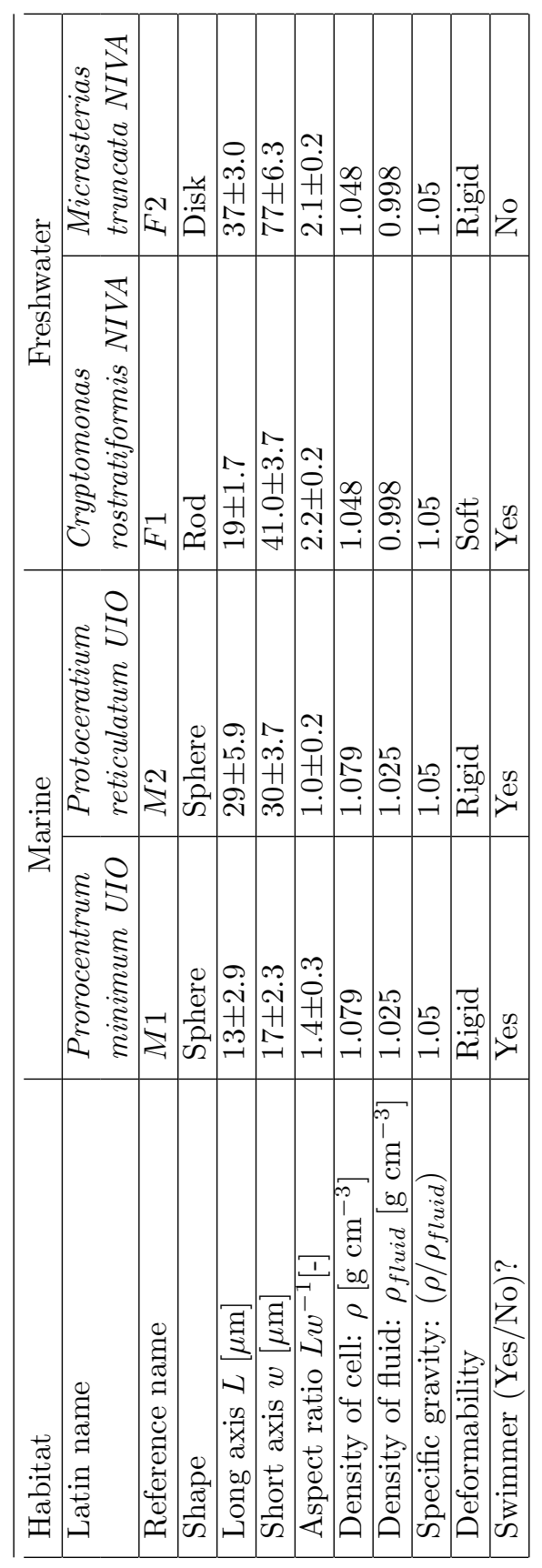

Table 1: Algal species. Dimensions are mean values \pm standard deviations. The cells are $5 \%$ heavier than their respective immersion medium, giving rise to weak inertial contributions. 


\begin{tabular}{|l|l|l|l|l|}
\hline Diameter $[\mu \mathrm{m}]$ & $21.02 \pm 2.01$ & $24.4688 \pm 2.2796$ & $32.4500 \pm 6.8877$ & $69.8538 \pm 3.6565$ \\
\hline $\mathrm{CR}[\mu \mathrm{m}]$ & $1.04 \pm 0.10$ & $1.08 \pm 0.10$ & $1.10 \pm 0.11$ & $1.42 \pm 0.14$ \\
\hline$Q[\mathrm{ml} / \mathrm{min}]$ & $32 \pm 0.03$ & $32 \pm 0.03$ & $18 \pm 0.02$ & $10 \pm 0.01$ \\
\hline Density of particle: $\rho\left[\mathrm{g} \mathrm{cm}^{-3}\right]$ & $1.00 \pm 0.01$ & $1.00 \pm 0.01$ & $1.00 \pm 0.01$ & $1.00 \pm 0.01$ \\
\hline Density of fluid: $\rho_{\text {fluid }}\left[\mathrm{g} \mathrm{cm}^{-3}\right]$ & 0.998 & 0.998 & 0.998 & 0.998 \\
\hline Specific gravity: $\left(\rho / \rho_{\text {fluid }}\right)$ & 1.00 & 1.00 & 1.00 & 1.00 \\
\hline Concentration $[$ by weight] & $1.7 \pm 2 \cdot 10^{-5}$ & $8.6 \pm 9 \cdot 10^{-5}$ & $86 \pm 9 \cdot 10^{-4}$ & $6.9 \pm 7 \cdot 10^{-5}$ \\
\hline
\end{tabular}

Table 2: Rigid spheres (polymer) and concentration ratios (CR) used for calibration and verification of the system. The listed values are mean values \pm standard deviations. The particles are neutrally buoyant, meaning that density differences do not influence the particles' inertia and the concentration ratios. 


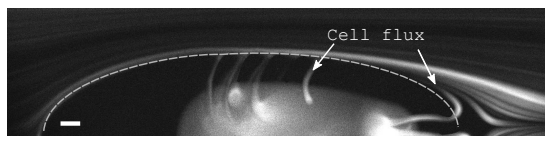

(a)

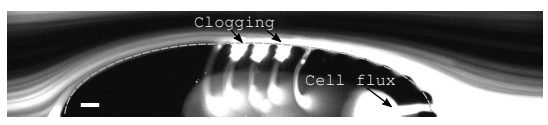

(c)

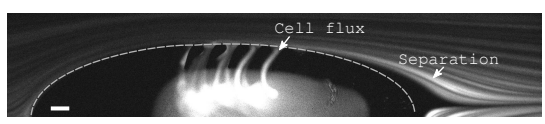

(b)

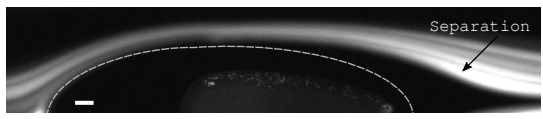

(d)

Figure 3: Concentration of sphere-like cells, M1 and M2. (a,b): Concentration of Prorocentrum minimum (M1): (a) High flux of cells through the slits due to low flow velocity and (b) separation by cross-streamline migration in the downstream region induced by inertia at high flow velocities $(Q=32 \mathrm{ml} / \mathrm{min})$, however a finite amount of cells slip through the slits and contaminate the permeate solution. (c,d): Enrichment of Protoceratium reticulatum (M2). (c) Unlike the case for test spheres, an attempt to separate these sphere-like cells by migration along pillars leads to clogging and thus an increase in flow rate is required to induce inertia which is used for migration across streamlines (d). Broken line indicates wall position. Scale bar is $50 \mu \mathrm{m}$.

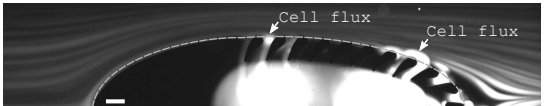

(a)

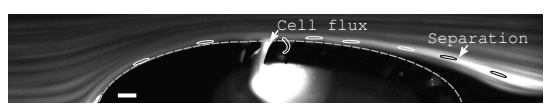

(b)

Figure 4: Concentration of the flexible, rod-like freshwater cell Cryptomonas rostratiformis (F1). (a) The flow rate is too low for separation from the filtrate stream, which is the employed method for concentration, and the result is a high flux of cells through the pillar gaps. (b) Separation by migration across streamlines at high flow velocity, especially in the downstream region, due to the high streamline curvature. However, there is a finite flux of cells in the upstream region because these flexible cells are deformed during impact with pillars instead of being deflected into the bulk flow by means of the golfballeffect. Broken line indicates wall position. Scale bar is $50 \mu \mathrm{m}$. 


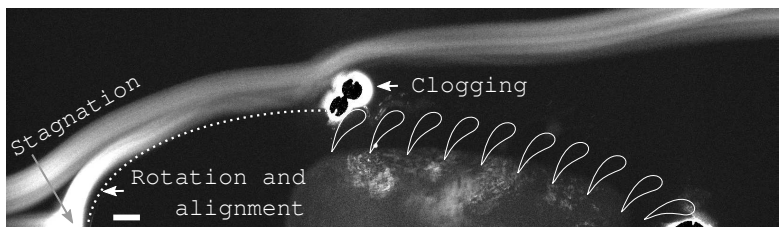

(a)

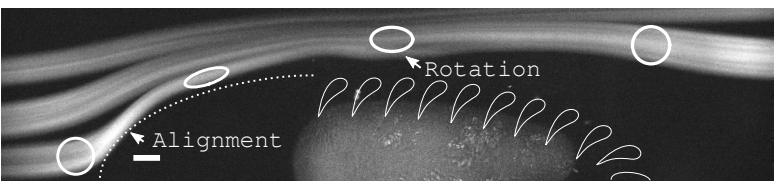

(b)

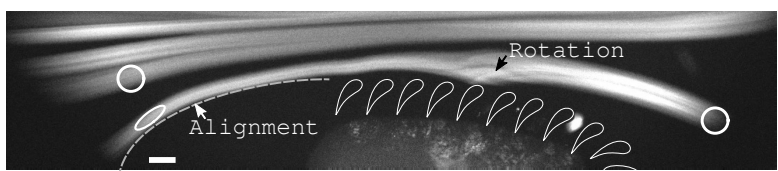

(c)

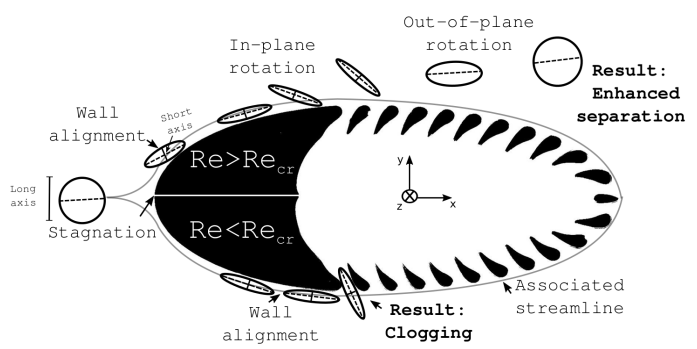

(d)

Figure 5: Separation of Micrasterias truncata (F2): (a) Attempt to separate particles by low-velocity rolling at $Q=10 \mathrm{ml} / \mathrm{min}$ results in clogging because the cells align with the wall near the stagnation point on the upstream bluff body. (b) Separation based on shortest diameter $(Q=18 \mathrm{ml} / \mathrm{min})$ : Successful separation, but since velocities are higher than the necessary to avoid clogging, the concentration efficiency is not maximized. (c) Separation by critical velocity $(Q=14 \mathrm{ml} / \mathrm{min})$. The cell rotation in the filter region prevents clogging. The broken line indicated the wall location. (d) Concentration principle used for disks: Separation with higher velocities (or Reynolds number $R e$ ) than necessary to avoid clogging, i.e. super-critical velocities, $R e>R e_{c r}$ (upper half), and clogging because velocities are lower than necessary to induce disk rotation which is required to avoid clogging, i.e. sub-critical velocities, $R e<R e_{c r}$ (lower half). The cell alignment near the stagnation point leads to clogging (lower half), while the in-plane cell rotation near the pillars prevents clogging (upper half). Scale bar is $50 \mu \mathrm{m}$. 


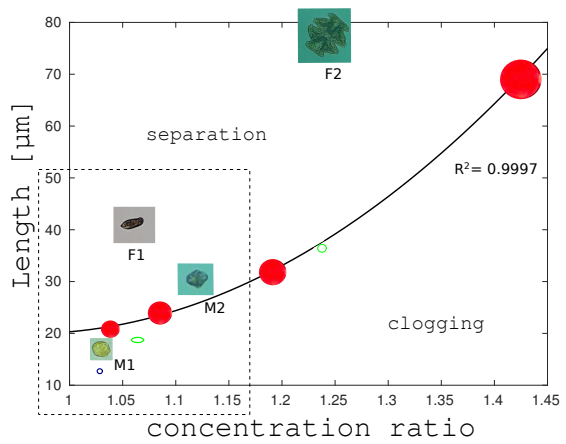

(a)

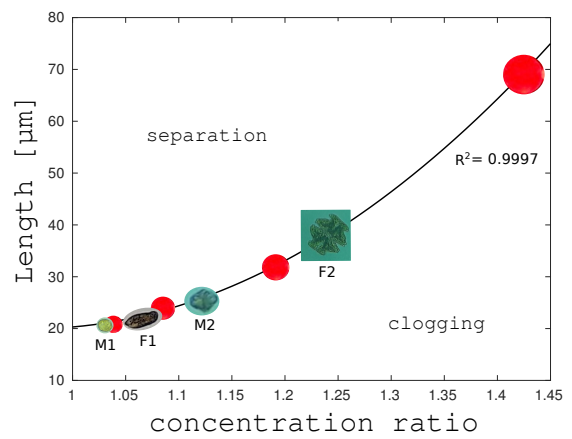

(c)

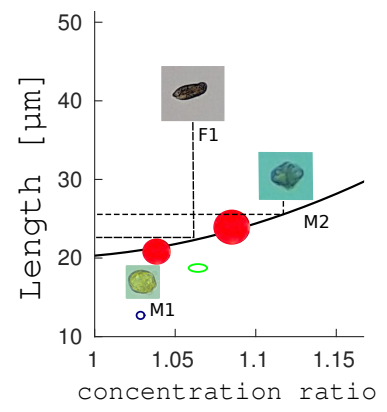

(b)

Figure 6: (a) Concentration ratio on the horizontal axis plotted against the particle dimensions (images indicate longest dimension, $L$, and circles are shortest dimension, $w)$. The red dots are the concentration ratios for the test spheres (polymer beads) and the solid line is a second order fit based on these results $\left(R^{2}=0.9997\right)$. (b) Zoom view shows how the 'separation diameter' $a_{H Y D}$ is found. (c) Algal species collapsed onto calibration curve for comparison with test spheres. 


\begin{tabular}{|c|l|l|l|l|}
\hline Name & CR & $w[\mu \mathrm{m}]$ & $L[\mu \mathrm{m}]$ & $a_{H Y D}[\mu \mathrm{m}]$ \\
\hline \hline M1 & 1.03 & 13 & 17 & 21.6 \\
\hline M2 & 1.11 & 29 & 30 & 25.6 \\
\hline F1 & 1.06 & 19 & 41 & 22.2 \\
\hline F2 & 1.21 & 37 & 77 & 37.5 \\
\hline
\end{tabular}

Table 3: Results of algae separation experiments, where $w$ is the cell thickness, $L$ is the cell length and $a_{H Y D}$ is the equivalent separation diameter of the algae cells if they were rigid spheres. 


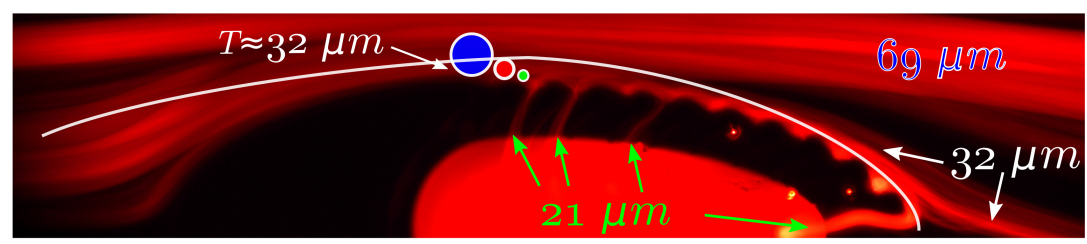

(a)

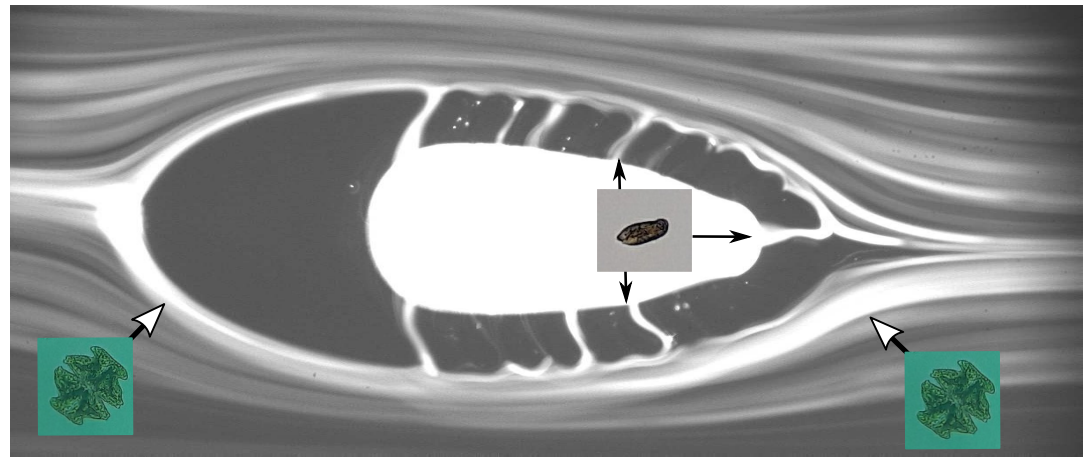

(b)

Figure 7: (a) Separation of spheres, where the solid line indicates the extent of filtrate flow layer, which is comparable in size to $32 \mu \mathrm{m}$ spheres. (b) Separation of complex algae; rod-like cells (F1) are separated from disk-like cells(F2)). In both (a) and (b), differences in degree of inertia are employed for separation. 


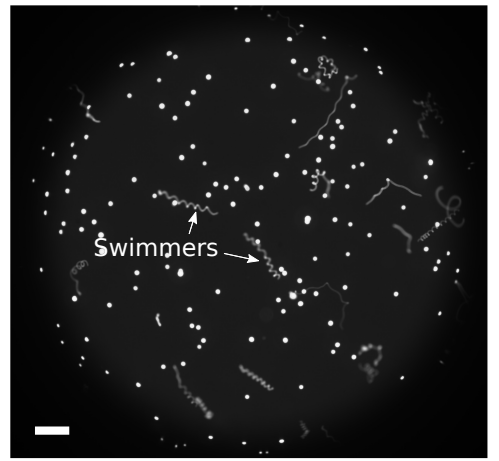

(a)

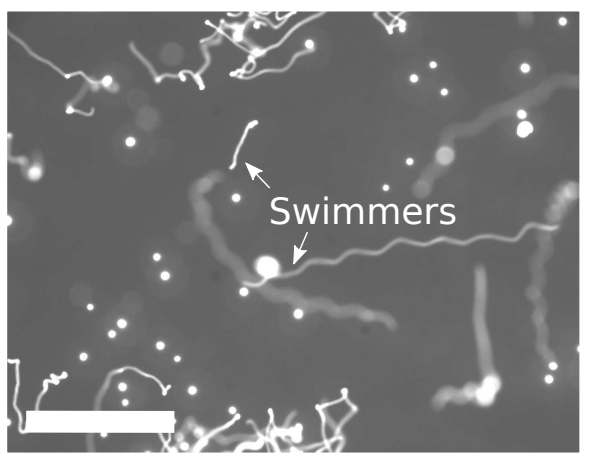

(b)

Figure 8: Long exposure images of swimming cells in drops deposited onto a microscope slide: (a) Trajectories of Protoceratium reticulatum (M2) in a $2 \mu \mathrm{l}$ drop viewed through a $4 \mathrm{x}$-objective clearly shows the helical swimming pattern. The speed is negligible compared to the speed of a cell in the flow field around the separation units, and thus the swimming does not influence the separation and concentration dynamics. (b) Long exposure image of swimming Protoceratium reticulatum (M2) in a $110 \mu \mathrm{l}$ drop viewed through a 10x-objective. Scale bar is $400 \mu \mathrm{m}$. Exposure time is 5 seconds. 


\begin{tabular}{|l|l|l|l|l|}
\hline Habitat & \multicolumn{2}{|c|}{ Marine } & \multicolumn{2}{c|}{ Freshwater } \\
\hline Latin name & $\begin{array}{l}\text { Prorocentrum } \\
\text { minimum UIO }\end{array}$ & $\begin{array}{l}\text { Protoceratium } \\
\text { reticulatum UIO }\end{array}$ & $\begin{array}{l}\text { Cryptomonas } \\
\text { rostratiformis NIVA }\end{array}$ & $\begin{array}{l}\text { Micrasterias } \\
\text { truncata NIVA }\end{array}$ \\
\hline Reference name & $M 1$ & M2 & F1 & F2 \\
\hline Swimmer (Yes/No)? & Yes & Yes & Yes & No \\
\hline Growth medium & IMR1/2, 25 PSU & IMR1/2,25 PSU & $20 \%$ Z8+vit & Z8 \\
\hline
\end{tabular}

Table 4: Algal species, additional properties 


\section{References}

Wisam Al-Faqheri, Tzer Hwai Gilbert Thio, Mohammad Ameen Qasaimeh, Andreas Dietzel, Marc Madou, and Ala'aldeen Al-Halhouli. Particle/cell separation on microfluidic platforms based on centrifugation effect: a review. Microfluidics and Nanofluidics, 21(6):102, May 2017. ISSN 1613-4990. doi: 10.1007/s10404-017-1933-4. URL https://doi.org/10.1007/s10404-017-1933-4.

Sandhya Babel and Satoshi Takizawa. Microfiltration membrane fouling and cake behavior during algal filtration. Desalination, 261(1):46 - 51, 2010. ISSN 0011-9164. doi: https://doi.org/10.1016/j.desal.2010.05.038. URL http://www.sciencedirect.com/science/article/pii/S001191641000353X.

Ana I. Barros, Ana L. Gonçalves, Manuel Simões, and José C.M. Pires. Harvesting techniques applied to microalgae: A review. Renewable and Sustainable Energy Reviews, 41:1489 - 1500, 2015. ISSN 1364-0321. doi: https://doi.org/10.1016/j.rser.2014.09.037. URL http://www.sciencedirect.com/science/article/pii/S1364032114008107.

Jason P. Beech, Stefan H. Holm, Karl Adolfsson, and Jonas O. Tegenfeldt. Sorting cells by size, shape and deformability. Lab Chip, 12:1048-1051, 2012. doi: 10.1039/C2LC21083E.

Chih-Chung Chen, Yu-An Chen, Yi-Ju Liu, and Da-Jeng Yao. A multilayer concentric filter device to diminish clogging for separation of particles and microalgae based on size. Lab Chip, 14:1459-1468, 2014. doi: 10.1039/C3LC51345A. URL http://dx.doi.org/10.1039/C3LC51345A.

J.P. Dijkshoorn, M.A.I. Schutyser, R.M. Wagterveld, C.G.P.H. Schroën, and R.M. Boom. A comparison of microfiltration and inertiabased microfluidics for large scale suspension separation. Separation and Purification Technology, 173:86 - 92, 2017. ISSN 13835866. doi: https://doi.org/10.1016/j.seppur.2016.09.018. URL http://www.sciencedirect.com/science/article/pii/S1383586616317099.

Tao Dong, Zhaochu Yang, Qianhua Su, Nhut Minh Tran, Eirik Bentzen Egeland, Frank Karlsen, Yulong Zhang, Matteo Joseph Kapiris, and Henrik Jakobsen. Integratable non-clogging microconcentrator based on counter-flow principle for continuous enrichment of caski cells sample. Microfluidics and Nanofluidics, 10(4):855-865, 2011. ISSN 1613-4990. doi: 10.1007/s10404-010-0717-x. URL http://dx.doi.org/10.1007/s10404-010-0717-x.

B. Edvardsen, F. Moy, and E. Paasche. Hemolytic activity in extracts of chrysochromulina polylepis grown at different levels of selenite and phosphate. In Toxic Marine Phytoplankton, pages 284-289. Elsevier, New York, 1990.

R.W. Eppley, R.W. Holmes, and J.D.H. Strickland. Sinking rates of marine phytoplankton measured with a fluorometer. J. Exp. Mar. Biol. Ecol., 1: 191-208, 1967. 
Sepehr Ghadami, Reza Kowsari-Esfahan, Mohammad Said Saidi, and Keykhosrow Firoozbakhsh. Spiral microchannel with stair-like cross section for size-based particle separation. Microfluidics and Nanofluidics, 21(7):115, Jun 2017. ISSN 1613-4990. doi: 10.1007/s10404-017-1950-3. URL https://doi.org/10.1007/s10404-017-1950-3.

Neus Godino, Felix Jorde, Daryl Lawlor, Magnus Jaeger, and Claus Duschl. Purification of microalgae from bacterial contamination using a disposable inertia-based microfluidic device. Journal of Micromechanics and Microengineering, 25(8):084002, 2015. URL http://stacks.iop.org/0960-1317/25/i=8/a=084002.

Stefan H. Holm, Jason P. Beech, Michael P. Barrett, and Jonas O. Tegenfeldt. Separation of parasites from human blood using deterministic lateral displacement. Lab Chip, 11:1326-1332, 2011. doi: 10.1039/C0LC00560F. URL http://dx.doi.org/10.1039/COLC00560F.

Birgitte K. Honsvall, Dag Altin, and Lucy J. Robertson. Continuous harvesting of microalgae by new microfluidic technology for particle separation. Bioresource Technology, 200:360 - 365, 2016. ISSN 0960-8524. doi: https://doi.org/10.1016/j.biortech.2015.10.046. URL http://www.sciencedirect.com/science/article/pii/S0960852415014492.

Kaitlyn Hood, Soroush Kahkeshani, Dino Di Carlo, and Marcus Roper. Direct measurement of particle inertial migration in rectangular microchannels. Lab Chip, 16:2840-2850, 2016. doi: 10.1039/C6LC00314A. URL http://dx.doi.org/10.1039/C6LC00314A.

Lotien Richard Huang, Edward C. Cox, Robert H. Austin, and James C. Sturm. Continuous particle separation through deterministic lateral displacement. Science, 304(5673):987-990, 2004. doi: 10.1126/science.1094567. URL http://www. sciencemag. org/content/304/5673/987. abstract.

Mingliang Jiang, Aaron D. Mazzeo, and German Drazer. Centrifuge-based deterministic lateral displacement separation. Microfluidics and Nanofluidics, 20(1):17, Jan 2016. ISSN 1613-4990. doi: 10.1007/s10404-015-1686-x. URL https://doi.org/10.1007/s10404-015-1686-x.

Youwei Jiang, Zhenming Yu, Xinglong Huang, Rifei Chen, Weiyuan Chen, Yifan Zeng, Chengqi Xu, Haodi Min, Nan Zheng, and Xing Cheng. A multilayer lateral-flow microfluidic device for particle separation. Microfluidics and Nanofluidics, 22(4):40, Mar 2018. ISSN 1613-4990. doi: 10.1007/s10404-0182053-5. URL https://doi.org/10.1007/s10404-018-2053-5.

D. Kamykowski, R. E. Reed, and G. J. Kirkpatrick. Comparison of sinking velocity, swimming velocity, rotation and path characteristics among six marine dinoflagellate species. Marine Biology, 113(2):319328, Jun 1992. ISSN 1432-1793. doi: 10.1007/BF00347287. URL https://doi.org/10.1007/BF00347287. 
Hyun Chul Kim, Jaewon Park, Younghak Cho, Hyunsoo Park, Arum Han, and Xing Cheng. Lateral-flow particle filtration and separation with multilayer microfluidic channels. Journal of Vacuum Science \& Technology B: Microelectronics and Nanometer Structures Processing, Measurement, and Phenomena, 27(6):3115-3119, 2009. doi: 10.1116/1.3258155. URL https://avs.scitation.org/doi/abs/10.1116/1.3258155.

J. Kotai. Instructions for preparation of modified nutrient solution Z8 for algae. Publication 8-11169. Norwegian Institute for Water Research, Blindern, Norway., 1972.

Andreas Lenshof and Thomas Laurell. Continuous separation of cells and particles in microfluidic systems. Chem. Soc. Rev., 39:1203-1217, 2010. doi: 10.1039/B915999C. URL http://dx.doi.org/10.1039/B915999C.

Liyu Liu, Kevin Loutherback, David Liao, David Yeater, Guillaume Lambert, Andre Estevez-Torres, James C. Sturm, Robert H. Getzenberg, and Robert H. Austin. A microfluidic device for continuous cancer cell culture and passage with hydrodynamic forces. Lab Chip, 10:1807-1813, 2010. doi: 10.1039/C003509B. URL http://dx.doi.org/10.1039/C003509B.

Kevin Loutherback, Kevin S. Chou, Jonathan Newman, Jason Puchalla, Robert H. Austin, and James C. Sturm. Improved performance of deterministic lateral displacement arrays with triangular posts. Microfluidics and Nanofluidics, 9(6):1143-1149, Dec 2010. ISSN 1613-4990. doi: 10.1007/s10404-010-0635-y. URL https://doi.org/10.1007/s10404-010-0635-y.

Julien Marchalot, Yves Fouillet, and Jean-Luc Achard. Multi-step microfluidic system for blood plasma separation: architecture and separation efficiency. Microfluidics and Nanofluidics, 17(1):167-180, Jul 2014. ISSN 1613-4990. doi: 10.1007/s10404-013-1296-4. URL https://doi.org/10.1007/s10404-013-1296-4.

M. Sneha Maria, B. S. Kumar, T. S. Chandra, and A. K. Sen. Development of a microfluidic device for cell concentration and blood cell-plasma separation. Biomedical Microdevices, 17(6):115, 2015. ISSN 1572-8781. doi: 10.1007/s10544-015-0017-z. URL http://dx.doi.org/10.1007/s10544-015-0017-z.

M. Sneha Maria, T. S. Chandra, and A. K. Sen. Capillary flowdriven blood plasma separation and on-chip analyte detection in microfluidic devices. Microfluidics and Nanofluidics, 21(4):72, Mar 2017. ISSN 1613-4990. doi: 10.1007/s10404-017-1907-6. URL https://doi.org/10.1007/s10404-017-1907-6.

J. McGrath, M. Jimenez, and H. Bridle. Deterministic lateral displacement for particle separation: a review. Lab Chip, 14:4139-4158, 2014 . doi: 10.1039/C4LC00939H. URL http://dx.doi.org/10.1039/C4LC00939H. 
Tomoki Morijiri, Masumi Yamada, Toshikatsu Hikida, and Minoru Seki. Microfluidic counterflow centrifugal elutriation system for sedimentationbased cell separation. Microfluidics and Nanofluidics, 14(6):1049-1057, Jun 2013. ISSN 1613-4990. doi: 10.1007/s10404-012-1113-5. URL https://doi.org/10.1007/s10404-012-1113-5.

E. J. Mossige, A. Jensen, and M. M. Mielnik. An experimental characterization of a tunable separation device. Microfluidics and Nanofluidics, 20 (12):160, 12 2016. ISSN 1613-4982. doi: 10.1007/s10404-016-1826-y. URL http:https://dx.doi.org/10.1007/s10404-016-1826-y.

E. J. Mossige, A. Jensen, and M. M. Mielnik. Separation and concentration without clogging using a high-throughput tunable filter. Phys. Rev. Applied, 9:054007, May 2018. doi: 10.1103/PhysRevApplied.9.054007. URL https://link.aps.org/doi/10.1103/PhysRevApplied.9.054007.

Jeonghun Nam, Yong Shin, Justin Kok Soon Tan, Ying Bena Lim, Chwee Teck Lim, and Sangho Kim. High-throughput malaria parasite separation using a viscoelastic fluid for ultrasensitive pcr detection. Lab Chip, 16:2086-2092, 2016. doi: 10.1039/C6LC00162A. URL http://dx.doi.org/10.1039/C6LC00162A.

Olgae. Comprehensive report on attractive algae product opportunities. http://www.oilgae.com/ref/report/non-fuel-algae-products.html, 2015.

Amit Prabhakar, Y. V. Bala Varun Kumar, Siddhartha Tripathi, and Amit Agrawal. A novel, compact and efficient microchannel arrangement with multiple hydrodynamic effects for blood plasma separation. Microfluidics and Nanofluidics, 18(5):995-1006, May 2015. ISSN 1613-4990. doi: 10.1007/s10404-014-1488-6. URL https://doi.org/10.1007/s10404-014-1488-6.

P. Sajeesh and Ashis Kumar Sen. Particle separation and sorting in microfluidic devices: a review. Microfluidics and Nanofluidics, 17(1):152, Jul 2014. ISSN 1613-4990. doi: 10.1007/s10404-013-1291-9. URL https://doi.org/10.1007/s10404-013-1291-9.

Sari Sugaya, Masumi Yamada, and Minoru Seki. Observation of nonspherical particle behaviors for continuous shape-based separation using hydrodynamic filtration. Biomicrofluidics, 5(2):024103, 2011. doi: 10.1063/1.3580757. URL http://dx.doi.org/10.1063/1.3580757.

Majid Ebrahimi Warkiani, Andy Kah Ping Tay, Bee Luan Khoo, Xu Xiaofeng, Jongyoon Han, and Chwee Teck Lim. Malaria detection using inertial microfluidics. Lab Chip, 15:1101-1109, 2015. doi: 10.1039/C4LC01058B. URL http://dx.doi.org/10.1039/C4LC01058B. 